\title{
SÍNDROME DEL COMEDOR NOCTURNO: NUEVO PLAN DE CUIDADOS DESDE LA ENFERMERÍA
}

\section{ISABEL MIGUEL ANDRÉS}

Enfermera. Centro de Salud Embarcaciones. Tres Cantos (Madrid).

\section{RESUMEN}

El síndrome del comedor nocturno se clasificó recientemente como un trastorno de la conducta alimentaria y se configuró como un síndrome en el que se producía una cronodisrupción del organismo, dando lugar a alteraciones de los biorritmos hormonales y de la nutrición. Aunque desconocido por múltiples profesionales de la salud, se ha considerado un trastorno singular que parece estar estrechamente relacionado con otros trastornos prevalentes del s. XXI como la obesidad, la ansiedad o la depresión, donde la enfermería desempeña una relevante función asistencial. El objetivo de esta revisión es, tras dar a conocer sus principales características, analizar el rol enfermero en el diagnóstico y tratamiento de este síndrome, dentro del ámbito de la atención comunitaria. Para la consecución de tal fin, se diseña un plan de cuidados de enfermería adaptado al paciente "comedor nocturno» y, tras ello, se destaca la gran necesidad de investigarlo en España para estimar su prevalencia real y verificar la eficacia de los cuidados enfermeros propuestos.

Palabras clave: atención de enfermería, fenómenos cronobiológicos, trastornos de la alimentación y de la ingestión de alimentos, obesidad y síndrome del comedor nocturno.

\section{INTRODUCCIÓN}

La alimentación no solo es una necesidad fisiológica. Los hábitos y conductas alimentarias de un individuo o grupo social vienen condicionados por la cultura, las normas y los principios de la sociedad a la que pertenece ${ }^{1}$. La configuración de la

Correspondencia: Isabel Miguel Andrés

Correo electrónico: isabel.miguela.94@gmail.com sociedad occidental del siglo XXI, marcada por fenómenos como la globalización, la publicidad y el marketing, unidos a los desorganizados y estresantes estilos de vida de la población urbana², ha contribuido al aumento de la prevalencia de trastornos como la obesidad ${ }^{1}$ y a la aparición de nuevos trastornos de la conducta alimentaria (TCA), entre los que se encuentra el síndrome del comedor nocturno (NES; por las siglas en inglés de: night-eating syndrome $)^{2}$. 
El NES, conocido divulgativamente como atracón nocturno, es un trastorno singular cuya clínica principal es la hiperfagia nocturna y las ingestiones nocturnas, manifestaciones que parecen ligarse a un proceso de cronodisrupción o interrupción de los ritmos circadianos de diversas hormonas y biorritmos de la nutrición de nuestro organismo. Además, tiende a acompañarse de alteraciones en el estado de ánimo y del sueño ${ }^{3}$.

La primera definición de dicho síndrome fue propuesta por Stunkard en 1955. No obstante, su investigación no comenzó a consolidarse hasta finales de la década de 1980 y, desde entonces, su conceptualización se ha caracterizado por la falta de precisión y disparidad 4 . En el año 2013, fue incluido como etiqueta diagnóstica en la quinta edición del Manual diagnóstico y estadístico de los trastornos mentales (DSM-5)5 (tabla 1 ).

En la actualidad, diversos estudios estiman la prevalencia en la población general de entre el 1,1 y el $1,5 \%{ }^{6}$. Este porcentaje aumenta en casos de obesidad (6-16\%); trastorno por atracón (BED; por las siglas en inglés de: binge eating disorder) (15-44\%); bulimia (9-47\%); trastornos del estado de ánimo (TEA), como los depresivos (33\%) o ansiosos (29\%); trastornos psicóticos $(14,4 \%)$ y disruptivos $(7,2 \%)^{6,7}$.
Los factores de riesgo para el NES han sido investigados a través de diversos estudios transversales publicados por autores como Saraçli et al. ${ }^{7}$ o Runfola et al. ${ }^{8}$. Entre los factores que identificaron, se encuentran: altos niveles de estrés o ansiedad, patrones del sueño o de la alimentación desorganizados, antecedentes personales de TCA y TEA, trastornos de la imagen corporal, obesidad, uso concomitante de sustancias y conductas impulsivas. También se correlacionaron factores sociodemográficos como: aumento de la edad, sexo masculino y problemas socioeconómicos ${ }^{6,9}$.

A día de hoy, los estudios de investigación revelan la necesidad de incrementar el conocimiento científico acerca del síndrome revisado, ya que la carencia de teorías firmes ha provocado que frecuentemente los propios profesionales de la salud no lo contemplen como un diagnóstico específico ${ }^{6}$. Así lo reflejaron Vandereycken y su equipo ${ }^{10}$ al revelar que el 43,2\% de una muestra de profesionales formados en TCA (tamaño de la muestra $=111$ ) desconocía el síndrome, y que el 38,7\% lo identificaron como una variante de otro TCA. Como mostraron Goncalves et al. ${ }^{11}$, la consecuencia de este desconocimiento repercute directamente sobre la calidad del diagnóstico y puede dar lugar a la implementación de tratamientos inadecuados y, por lo tanto, a

Tabla 1. Criterios diagnósticos del síndrome del comedor nocturno

Episodios recurrentes:

- Consumo excesivo de alimentos después de cenar.

- Ingestas durante la noche tras despertarse.

Existe consciencia y recuerdo de la ingesta nocturna.

Trastorno asociado a ansiedad y altos niveles de estrés. Causa disfuncionalidad psicosocial.

No es atribuible a influencias externas, como modificaciones en el ciclo de sueño-vigilia del individuo o a normas sociales.

No es un trastorno secundario al abuso o dependencia de sustancias tóxicas, problemas de salud orgánicos u otros trastornos mentales ni efecto adverso de un tratamiento farmacológico. 


\section{Los propios profesionales de la salud no lo contemplan como un diagnóstico específico.}

la insatisfacción por parte de los pacientes «comedores nocturnos».

Ciertamente, por las características que conforman el NES, parece que el ámbito más adecuado para realizar su detección y posterior seguimiento sería bajo un contexto de atención comunitaria ${ }^{12}$; tanto desde las consultas de atención primaria (AP) como desde los centros de salud mental (CSM). En ambos ámbitos, la profesión enfermera desempeña un relevante rol asistencial.

En la literatura científica, no consta documentación referente a los cuidados enfermeros dirigidos específicamente a los pacientes «comedores nocturnos». No obstante, el síndrome objeto de estudio se clasifica como un subtipo de TCA $^{5}$ y, por lo tanto, los planes de cuidados y protocolos diseñados para casos de TCA en general podrían guardar gran similitud con los cuidados enfermeros específicos aplicables a un paciente con NES. Por otro lado, la obesidad se ha definido como uno de los principales trastornos comórbidos del NES, observándose que ciertas conductas características de un comedor nocturno pueden disminuir considerablemente la adherencia terapéutica ante un tratamiento para la obesidad ${ }^{13}$.

El objetivo general de esta revisión es analizar el papel que puede llegar a tener la enfermería en una detección precoz y en el seguimiento del paciente afectado por el NES en el contexto de la atención comunitaria.

\section{METODOLOGÍA}

La revisión narrativa se ha realizado a través de búsqueda bibliográfica en las bases de datos de cien- cias de la salud: CINAHL, Cochrane, CUIDEN, Dialnet, PubMed, SciELO, Scopus y Web of Science (WoS). También se consultaron otras fuentes como: webs oficiales del Instituto Nacional de Estadística (INE), del Ministerio de Sanidad, Servicios Sociales e Igualdad (MSSSI), del Instituto Nacional de Salud Mental (NIMH) estadounidense y de la Sociedad Española de Nutrición (SEN); la base de datos NNNConsult y Google Scholar.

Las palabras clave seleccionadas fueron: "chronobiology phenomena», "feeding and eating disorders», "night eating syndrome», "nursing care» $y$ "obesity». De ellos, el único término que ha sido redactado como «lenguaje libre» es «night eating syndrome». Los operadores empleados fueron: «booleanos: AND, OR» y «de proximidad: WITH». A su vez, los filtros empleados fueron: "journal article, reviews \& practise guideline», "free full text», "from 2009 to 2016», "English \& Spanish», "all adults» $y$ «humans». Tras la búsqueda bibliográfica en las bases de datos citadas, se hallaron alrededor de 250 publicaciones en total, de las cuales, se escogieron 37 para elaborar la revisión. En su mayoría, eran artículos originales y revisiones narrativas. Por otra parte, en referencia a las consultas realizadas en las webs oficiales mencionadas, se seleccionaron siete referencias.

\section{RESULTADOS}

\section{Etiología}

EI NES se ha configurado como un trastorno multicausal. Aunque se desconoce su etiología exacta ${ }^{6}$, el modelo biopsicosocial de Stunkard ${ }^{14}$, publicado en el año 2009, reveló que los factores causales que inciden sobre el desarrollo de la enfermedad podrían ser tanto de origen orgánico como genético o psicosocial.

A nivel orgánico, en el NES, parece tener lugar un proceso de cronodisrupción, como consecuencia de la desregulación tanto exógena como endógena del sistema circadiano humano $0^{9,15,16}$ (fig. 1). 
De acuerdo con la figura 1, se produce una alteración de los sincronizadores externos (ingesta/ayuno) al retrasarse los horarios de las comidas. A su vez, a nivel endógeno, se ha observado un retraso del ritmo habitual en la secreción cíclica de la melatonina y el cortisol. Dichas disrupciones quedan estrechamente relacionadas con otras alteraciones neuroendocrinas y metabólicas del NES descritas por autores como Vander Wal9: a nivel hormonal, el retraso en el ciclo de la leptina y la aceleración del bio- rritmo de la grelina parecen incidir positivamente sobre un aumento del estrés fisiológico y del apetito. Por otra parte, entre los comedores nocturnos, también se ha observado una discordancia entre los ciclos naturales de la glucosa-insulina y un retraso en el ritmo del metabolismo basal de carbohidratos y lípidos.

Otra teoría biológica ligada con la desregulación de estos ritmos circadianos y el aumento del apetito es la disfunción del sistema serotoninérgico6,14.

Figura 1. Organización y funcionamiento del sistema circadiano en humanos.

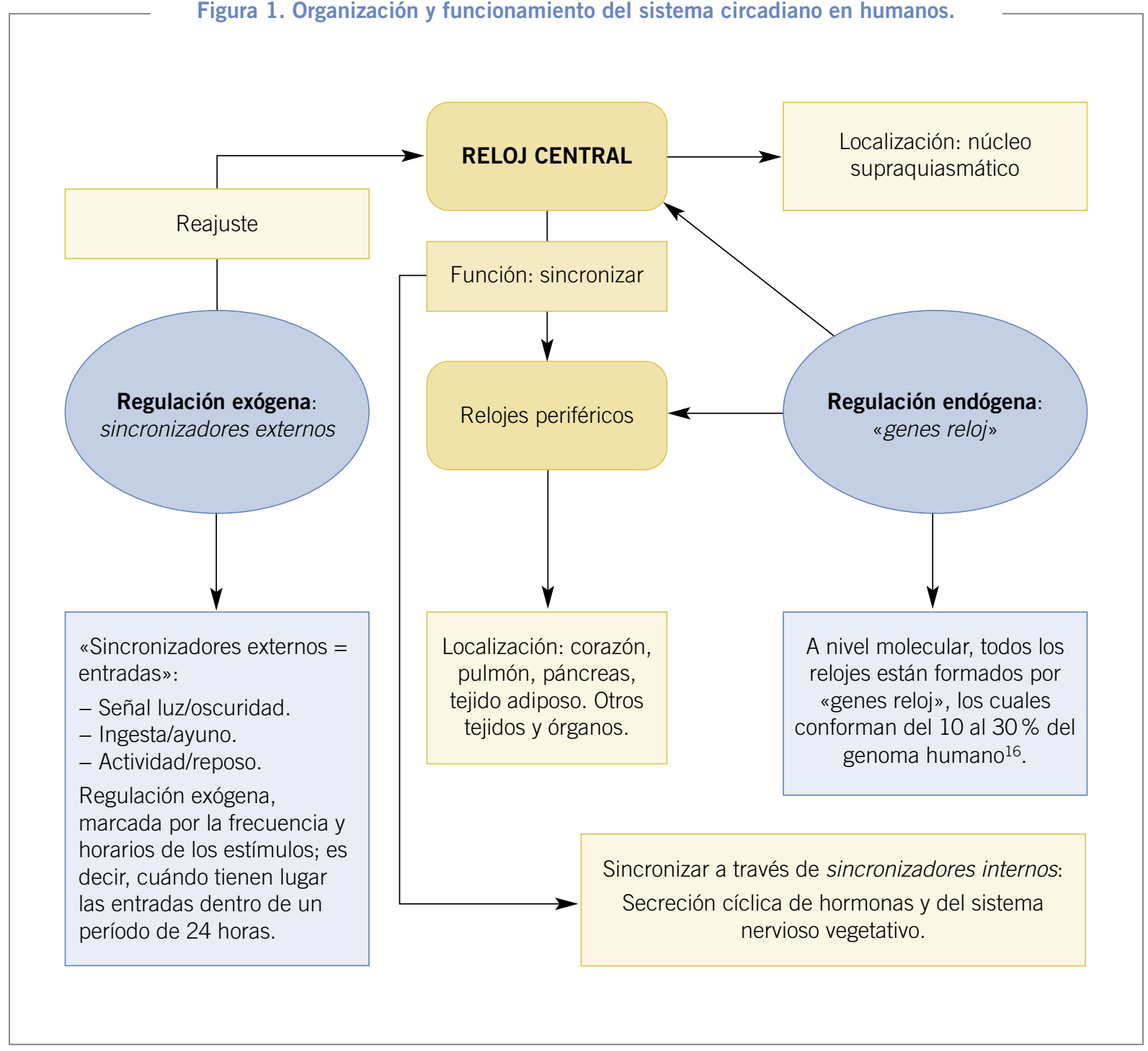


A nivel genético, no se han publicado a día de hoy estudios concluyentes, aunque diversas investigaciones reconocen la implicación de una base genética en el desarrollo de la enfermedad ${ }^{17}$. Por último, a nivel psicosocial, se han enfatizado factores como la vivencia de eventos vitales estresantes, el empleo de técnicas de afrontamiento inefectivas ${ }^{14}$ y un estilo de vida marcado por un cronotipo vespertino ${ }^{16}$.

\section{Comorbilidad}

\section{Obesidad}

La relación entre el NES y la obesidad ha sido una cuestión ampliamente estudiada3,6,17. Aunque todavía se desconoce a ciencia cierta qué tipo de causalidad guardan ambas enfermedades ${ }^{17}$, parecen evidenciarse ciertos factores que inciden positivamente sobre dicha correlación como: el aumento de la edad —especialmente, a partir de los 30 años $^{18}$ —, las ingestas emocionales ${ }^{19}$ y que las calorías ingeridas durante la noche son metabolizadas de manera menos eficiente por el organismo ${ }^{19}$.

\section{Trastorno por atracón}

EI NES se ha llegado a considerar como un subtipo de BED ${ }^{6}$. Por ello, se han diseñado las bases fundamentales para establecer un diagnóstico diferencial, las cuales se fundamentan en: la cantidad de alimentos ingeridos por episodio (en el NES, puede objetivarse simplemente como un picoteo), el momento de la ingesta (en el NES, solo es durante la noche) y la actitud frente a la comida (mientras que, en el atracón, se experimenta vergüenza y culpabilidad, en el NES, se puede concebir como un método para conciliar el sueño) $)^{6,8}$.

\section{Trastornos del estado de ánimo y del sueño}

Kucukgoncu et al. ${ }^{20}$ pusieron de manifiesto como los pacientes que compartían la depresión y el NES presentaban mayor gravedad de la clínica depresiva y ansiosa, y peor calidad del sueño.

\section{El síndrome del comedor nocturno se ha configurado como un trastorno multicausal, aunque se desconoce su etiología exacta.}

Por otra parte, se ha observado que, aunque el biorritmo sueño-vigilia parece permanecer inaltera$\mathrm{do}^{3,14}$, tanto el insomnio de inicio como de mantenimiento tiende a estar presente entre los pacientes «comedores nocturnos»6,9.

\section{Diagnóstico}

El diagnóstico es principalmente clínico y, actualmente, se cuenta con dos herramientas diagnósticas $^{6,9,18}$. Por un lado, el cuestionario autoadministrado de 17 ítems Night Eating Questionnaire (NEQ), el cual permite detectar el síndrome y valorar la gravedad de los síntomas; una puntuación superior a 30 puntos se considera criterio diagnóstico. Por otro lado, la entrevista semiestructurada en cuatro partes Night Eating Syndrome History and Invertory (NESHI), que debe ser realizada por un experto en TCA y permite establecer un diagnóstico preciso.

\section{Tratamiento}

El abordaje terapéutico del NES debe ser multidisciplinario para proporcionar a los pacientes una atención adecuada a nivel orgánico, nutricional y psicosocial. A su vez, los objetivos terapéuticos que deben plantearse son: conseguir unos hábitos saludables en la alimentación, estructurar los horarios de las comidas, controlar el peso e intervenir sobre los casos de TEA y el insomnio ${ }^{21}$.

\section{Tratamiento farmacológico}

A día de hoy, los fármacos empleados como primera línea de tratamiento en el NES son los antidepresivos inhibidores selectivos de la recaptación de serotonina (ISRS), especialmente, la sertralina y el 
escitalopram, ya que, en diversos estudios experimentales, se ha obtenido una considerable mejoría de la clínica. Como segunda línea de tratamiento, destacan los análogos de la melatonina (ramelteón y agomelatina), o el topiramato, como estabilizador del estado de ánimo ${ }^{6}$.

\section{Tratamiento no farmacológico}

- Terapia cognitivo-conductual (TCC): los «comedores nocturnos» tienden a presentar una serie de distorsiones cognitivas y alteraciones conductuales que pueden ser abordadas a través de la TCC 6,9,22. El pensamiento irracional más característico es la creencia de que «necesitan comer algo para poder dormirse de nuevo» 23 . A su vez, ello provoca que lleven a cabo una serie de conductas anormales, que refuerzan positivamente la clínica del síndrome. Por lo tanto, la TCC cuenta con un objetivo terapéutico específico: interrumpir el círculo vicioso que se inicia tras dicha distorsión cognitiva ${ }^{21}$. Para su consecución, este tipo de psicoterapia se estructura en siete pilares básicos: psicoeducación sobre la enfermedad, modificación de la alimentación a través de métodos como el diario dietético, técnicas de relajación, técnicas de reestructuración cognitiva, apoyo social, ejercicio físico y, por último, remisión de la medicación ${ }^{9,24}$.

- Otros tratamientos: terapia conductual (contratos de contingencia o técnicas de comportamiento ${ }^{22}$, terapia lumínica ${ }^{6}$ y cronoterapia, aquella dirigida a intervenir sobre los procesos de cronodisrupción, actuando a nivel de las alteraciones producidas en las entradas y salidas que regulan los ritmos circadianos ${ }^{16,17}$.

\section{El ámbito más adecuado para realizar su detección y posterior seguimiento sería bajo un contexto de atención comunitaria.}

\section{Relación del síndrome del comedor nocturno con la práctica clínica enfermera}

Baile et al. ${ }^{25}$ recogieron en su trabajo la descripción del rol enfermero adoptado con pacientes con TCA tanto en AP como en los CSM. En ambos ámbitos, su función asistencial se centra en el seguimiento a largo plazo. Los planes de cuidados y protocolos que se implementan están especialmente dirigidos a promover unos hábitos de vida saludables en relación con la alimentación, el sueño y el afrontamiento del estrés, así como a prevenir recaídas. Dichos objetivos son posibles mediante las siguientes intervenciones enfermeras (NIC): enseñanza del proceso de enfermedad y tratamientos, asesoramiento nutricional, control periódico del plan alimentario y apoyo emocional al paciente y familiares ${ }^{25,26}$. Además, desde los CSM, se pueden proponer otras intervenciones enfermeras más especializadas dirigidas a técnicas propias de la TCC como, por ejemplo: «NIC [6040] Terapia de relajación», «NIC [4700] Reestructuración cognitiva» 0 «NIC [4350] Manejo de la conducta»27-29.

A su vez, cabe hacer especial mención a algunos aspectos sobre el tratamiento enfermero para la obesidad. Larrañaga y García-Mayor ${ }^{13}$ manifestaron que la presencia de conductas patológicas que se encuadran dentro de determinados tipos de TCA, como el NES, suponían uno de los principales problemas en la adherencia al tratamiento de la obesidad. Nuevamente, se destaca la psicoeducación y otras técnicas de la TCC como medidas terapéuticas eficaces para mejorar los hábitos de alimentación respecto a qué, cómo, cuándo y dónde comer ${ }^{30}$.

\section{CONCLUSIONES}

En relación con la detección temprana del NES, se concluye que se puede identificar a los grupos de riesgo desde las consultas de enfermería, tanto de AP como de los CSM. A través de una valoración de enfermería por patrones funcionales de salud, 
es posible detectar problemas de salud asociados a los patrones de salud clave (fig. 2) ${ }^{27}$. Una vez identificada la población susceptible de padecer el trastorno, se les debería aplicar cuestionarios de detección específicos.

Por otra parte, tras la revisión de los resultados $12,24-26,29,30$, se considera que, desde la enfermería comunitaria, se puede realizar un abordaje integral e individualizado del paciente con NES. Por ello, en concordancia con el proceso de atención enfermero, se propone un plan de cuidados dirigido al NES (tabla 2)26-28,31,32.
Los cuidados propuestos persiguen principalmente los siguientes fines:

- A través de la psicoeducación, se pretende trabajar distintos ámbitos. En primer lugar, fomentar la conciencia de enfermedad, ya que, entre la población general, puede pasarse por alto la clínica patológica que define el trastorno. También enseñar unos hábitos alimentarios acordes con los biorritmos fisiológicos, incidiendo en la importancia de cuándo comer. Por último, educar en higiene del sueño, con la finalidad de normalizar los niveles de cortisol mediante intervencio-

Figura 2. Valoración enfermera teórica aplicada al síndrome del comedor nocturno. Patrones disfuncionales.

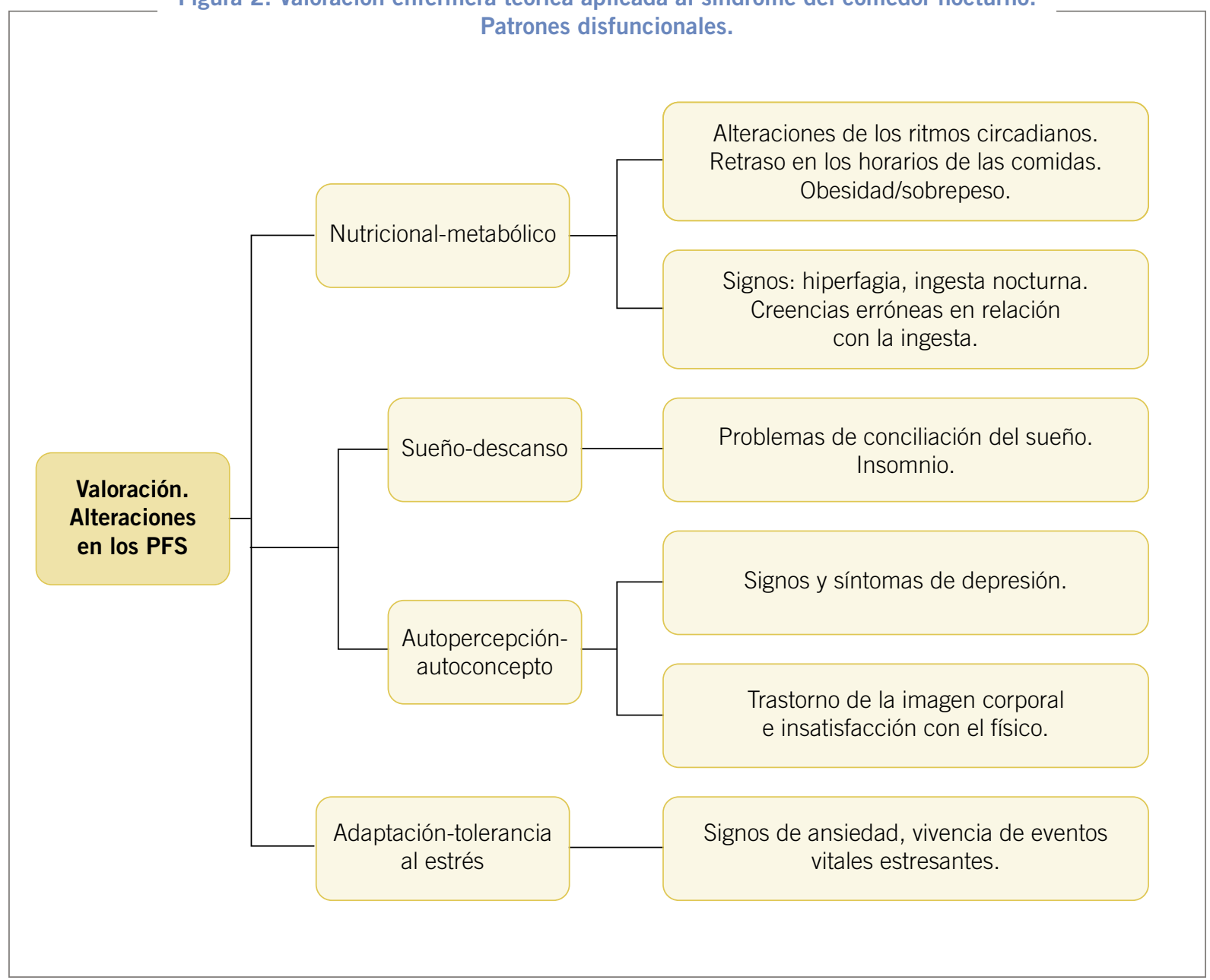

PFS: patrones funcionales de salud. 
Tabla 2. Esquema del diseño del plan de cuidados dirigido al síndrome del comedor nocturno

\begin{tabular}{|c|c|c|}
\hline Diagnóstico (NANDA) & Resultados (NOC) & Intervenciones (NIC) \\
\hline \multirow{2}{*}{ [78] Gestión ineficaz de la salud } & [1608] Control de síntomas & $\begin{array}{l}\text { [4470] Ayuda en la modificación } \\
\text { de sí mismo }\end{array}$ \\
\hline & [1601] Conducta de cumplimiento & $\begin{array}{l}\text { [5602] Enseñanza: proceso de } \\
\text { enfermedad }\end{array}$ \\
\hline [232] Obesidad & $\begin{array}{l}\text { [1627] Conducta de pérdida de } \\
\text { peso }\end{array}$ & [5246] Asesoramiento personal \\
\hline $\begin{array}{l}\text { [178] Trastorno del patrón del } \\
\text { sueño }\end{array}$ & [4] Sueño & [1850] Mejorar el sueño \\
\hline \multirow{2}{*}{$\begin{array}{l}\text { [118] Trastorno de la imagen } \\
\text { corporal }\end{array}$} & [1200] Imagen corporal & $\begin{array}{l}\text { [5400] Potenciación de la } \\
\text { autoestima }\end{array}$ \\
\hline & $\begin{array}{l}\text { [1302] Afrontamiento de } \\
\text { problemas }\end{array}$ & [4700] Reestructuración cognitiva \\
\hline \multirow{2}{*}{ [69] Afrontamiento ineficaz } & [1405] Autocontrol de impulsos & [4350] Manejo de la conducta \\
\hline & $\begin{array}{l}\text { [1302] Afrontamiento de } \\
\text { problemas }\end{array}$ & [5230] Mejorar el afrontamiento \\
\hline [146] Ansiedad & [1402] Autocontrol de ansiedad & [6040] Terapia de relajación \\
\hline
\end{tabular}

nes como la terapia de relajación 27,29,30. Por otra parte, para tratar los problemas derivados de la ansiedad, baja autoestima o afrontamiento ineficaz, la enfermería debe centrarse en aquellas intervenciones dirigidas a la puesta en práctica de técnicas de afrontamiento activas, así como al autocontrol de los impulsos y manejo de la conducta $^{22,26,27}$. Por último, intervenciones más específicas de la especialidad en salud mental son las técnicas de reestructuración cogniti$\mathbf{v a}^{27}$, dirigidas a modificar las creencias erróneas propias del paciente «comedor nocturno».

- Limitaciones del trabajo: en líneas generales, el NES se considera un síndrome aún desconocido ${ }^{6,10}$ y, además, concretamente, en España, parece haber quedado incluido en un «cajón de sastre» denominado trastornos de la conducta alimentaria no especificados (TCANE) ${ }^{33}$. Su des- conocimiento en nuestro país conlleva: ausencia de estudios de prevalencia, falta de aplicación de medidas de detección temprana y mayor probabilidad de error al realizar un diagnóstico diferencial. Además, en las guías de práctica clínica sobre TCA, no se describe un plan de cuidados estandarizado que identifique los problemas prevalentes y más característicos de estos pacientes.

- Propuestas de investigación y mejoras en la práctica clínica enfermera: se propone la elaboración de futuros estudios de prevalencia para determinar su incidencia en España. Asimismo, se recomienda estandarizar el uso de las herramientas de detección precoz en el síndrome, como el $N E Q$, para aplicar a la población de riesgo.

En el presente trabajo, se elabora un plan de cuidados estandarizado teórico. Sería interesante la 
implementación de dicho plan desde las consultas de enfermería de AP y de los CSM a través de estudios experimentales para comprobar su eficacia.

\section{AGRADECIMIENTOS:}

Mi más sincero agradecimiento a Isabel Calvo Viñuela, enfermera de la Unidad de Obesidad, Endocrinología y Nutrición del Hospital Universitario la Paz de Madrid, por introducirme en el presente tema de investigación, animarme a publicarlo y por sus consejos y apoyo durante este proceso.

\section{BIBLIOGRAFÍA}

1. Valera Moreiras G, Serrano Iglesias M, Alonso Aperte E, García González A, Anchón Tuñón M. Alimentación y sociedad en la España del siglo XXI. Madrid: Fundación Mapfre; 2015.

2. Sánchez-Barbudo A. Avances en medicina del adolescente: trastornos de la conducta alimentaria del S. XXI. Voxpaed. 2010;17(1):19-27.

3. Cleator J, Abbott J, Judd P, Sutton C, Wilding JP. Night eating syndrome: implications for severe obesity. Nutr Diabetes. 2012;2:e44.

4. Lundgren JD, McCune A, Spresser C, Harkins P, Zolton $L$, Mandal K. Night eating patterns of individuals with eating disorders: implications for conceptualizing the night eating syndrome. Psychiatry Res. 2011;186(1): 103-8.

5. American Psychiatric Association. Diagnostic and statistical manual of mental disorders. Fifth Edition (DSM5). Arlington: American Psychiatric Association; 2013.

6. Kucukgoncu S, Midura M, Tek C. Optimal management of night eating syndrome: challenges and solutions. Neuropsychiatr Dis Treat. 2015;11:751-60.

7. Saraçlı Ö, Atasoy N, Akdemir A, Güriz O, Konuk N, Sevinçer GM, et al. The prevalence and clinical features of the night eating syndrome in psychiatric outpatient population. Compr Psychiatry. 2015;57: 79-84.

8. Runfola CD, Allison KC, Hardy KK, Lock J, Peebles R. Prevalence and clinical significance of night eating syndrome in university students. J Adolesc Health. 2014;55(1):41-8.

9. Vander Wal JS. Night eating syndrome: a critical review of the literature. Clin Psychol Rev. 2012;32(1):49-59.

10. Vandereycken W. Media hype, diagnostic fad or genuine disorder? Professionals' opinions about night eating syndrome, orthorexia, muscle dysmorphia, and emetophobia. Eat Disord. 2011;19(2):145-55.
11. Goncalves MD, Moore RH, Stunkard AJ, Allison KC. The treatment of night eating: the patient's perspective. Eur Eat Disord Rev. 2009;17(3):184-90.

12. Sim LA, McAlpine DE, Grothe KB, Himes SM, Cockerill $R G$, Clark MM. Identification and treatment of eating disorders in the primary care setting. Mayo Clin Proc. 2010;85(8):746-51.

13. Larrañaga Vidal A, García-Mayor RV. Alta frecuencia de trastornos de la conducta alimentaria inespecíficos en personas obesas. Nutr Hosp. 2009;24(6):661-6.

14. Stunkard AJ, Allison KC, Lundgren JD, O'Reardon JP. A biobehavioural model of the night eating syndrome. Obes Rev. 2009;10 Suppl 2:69-77.

15. Garaulet Aza M. La cronobiología, la alimentación y la salud. Mediterr Econ. 2015;27:101-22.

16. Gómez-Abellán P, Madrid JA, Ordovás JM, Garaulet M. Aspectos cronobiológicos de la obesidad y el síndrome metabólico. Endocrinol Nutr. 2012;59(1):50-61.

17. Gallant AR, Lundgren J, Drapeau V. The night-eating syndrome and obesity. Obes Rev. 2012;13(6):528-36.

18. Meule A, Allison KC, Brähler E, de Zwaan M. The association between night eating and body mass depends on age. Eat Behav. 2014;15(4):683-5.

19. Meule A, Allison KC, Platte P. Emotional eating moderates the relationship of night eating with binge eating and body mass. Eur Eat Disord Rev. 2014;22(2):147-51.

20. Kucukgoncu S, Tek C, Bestepe E, Musket C, Guloksuz S. Clinical features of night eating syndrome among depressed patients. Eur Eat Disord Rev. 2014;22(2): 102-8.

21. Costa MB, Stein AT, Fernandes Moça Trevisani V, Valente O, Harb A, Melnik T. Pharmacological and psychosocial interventions for night eating syndrome in adults (Protocol). Cochrane Database Syst Rev. 2015; 4:CD011667.

22. Allison KC, Tarves EP. Treatment of night eating syndrome. Psychiatr Clin North Am. 2011;34(4):785-96.

23. Vinai $P$, Cardetti S, Studt S, Carpegna G, Ferrato N, Vallauri $P$, et al. Clinical validity of the descriptor "presence of a belief that one must eat in order to get to sleep» in diagnosing the night eating syndrome. Appetite. 2014;75:46-8.

24. Vander Wal JS, Maraldo TM, Vercellone AC, Gagne DA. Education, progressive muscle relaxation therapy, and exercise for the treatment of night eating syndrome. A pilot study. Appetite. 2015;89:136-44.

25. Baile J, Gil-Ezquerra R, Escalada-Abraham A. El papel de la enfermería en atención de pacientes con trastornos del comportamiento alimentario. Pulso. 2015;82: 30-3.

26. Nieto E, Casado A, García C, Peláez L. Guía de cuidados de enfermería familiar y comunitaria en los trastor- 
nos de la conducta alimentaria. 2. ${ }^{a}$ ed. España: FAECAP; 2009.

27. Caminero Luna P, Castelo Sardina C. Manual de procedimientos de enfermería de salud mental comunitaria en la comunidad de Madrid: planificación estratégica, comisión consultiva de cuidados de enfermería 20102014. Oficina Regional de Coordinación de Salud Mental. Madrid: Salud Madrid; 2012.

28. Bulechek G, Butcher H, Dochterman J, Wagner C. Clasificación de Intervenciones de Enfermería (NIC). 6. ${ }^{a}$ ed. Madrid: Elsevier; 2013.

29. Valera MR, Coletas J, Ramírez MA, Gracia A, Valera AB, Marín MJ. Trastornos del sueño y trastornos de la conducta alimentaria. Relajación con risoterapia. Estudio piloto. Rev Enferm Salud Ment. 2016;(3):5-12.
30. Torres V, Castro Sánchez AM, Matarán Peñarocha GA, Lara Palomo I, Aguilar Ferrándiz ME, Moreno Lorenzo C. Beneficios de la terapia cognitivo-conductual y la presoterapia en pacientes obesos: ensayo clínico aleatorizado. Nutr Hosp. 2011;26(5):1018-24.

31. Herdman H, Kamitsuru S. NANDA Internacional. Diagnósticos enfermeros. Definiciones y clasificación

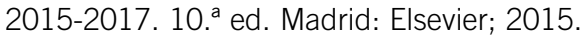

32. Moorhead S, Johnson M, Maas M, Swanson E. Clasificación de resultados de enfermería (NOC). 5. ${ }^{a}$ ed. Madrid: Elsevier; 2013.

33. Peláez Fernández MA, Raich Escursell RM, Labrador Encinas FJ. Trastornos de la conducta alimentaria en España: revisión de estudios epidemiológicos. Rev Mex Trastor Aliment. 2010;1(1):62-75. 